\title{
Aprendizaje de traslaciones en el plano fundamentado en el modelo de Van Hiele, mediado por Geogebra
}

\author{
Learning of transfers in the plan based on the Van Hiele model, \\ mediated by geogebra.
}

Aprendizagem de traduções no plano baseado no modelo de Van Hiele, mediado por geogebra.

Gonzalo Cáceres Bautista Licenciado en matemática e informática educativa Universidad Autónoma de Bucaramanga gcaceres241@unab.edu.co

\section{Resumen}

En el artículo se analiza el proceso de aprendizaje de las traslaciones en el plano tomando el modelo de Van Hiele e integrando el software geogebra, en los estudiantes de séptimo grado en el Centro Educativo Rural Sucre del municipio de Mutiscua, Colombia. Estos estudiantes han mostrado bajos resultados en las pruebas saber y entre las sugerencias que da el ministerio en el índice sintético de calidad ISCE está: fortalecer el componente geométrico y en particular las trasformaciones en el plano. La investigación fue cualitativa y en particular investigación acción. Se aplicó un pretest con el fin de determinar el nivel de aprendizaje de los estudiantes; luego, se diseñaron tres proyectos de aula divididos en 10 sesiones de trabajo planteadas acorde con las fases del modelo de Van Hiele y utilizando Geogebra como herramienta TIC (tecnologías de la información y comunicación). Al finalizar se evaluó su alcance el por medio de un postest. En el proceso se analizó el aprendizaje y algunas de sus características. El modelo de Van Hiele facilitó el aprendizaje de las traslaciones en el plano y permitió hacer una evaluación permanente del proceso de aprendizaje. Incluir herramientas de TIC permitió que los estudiantes realizaran el paso por la fase de integración de una forma práctica y agradable. Se deben diseñar las actividades basadas en el contexto y fomentar el trabajo en equipo. 
Palabras Clave: Aprendizaje, Traslaciones en el plano, Modelo de Van Hiele, Geogebra.

\begin{abstract}
The article analyzes the learning process of the translations in the plane using the Van Hiele model and integrating the software geogebra, in the students of seventh degree in the Rural Education Center Sucre of the municipality of Mutiscua, Colombia. These students have shown low results in the Pruebas Saber and among the suggestions given by the Ministry in the synthetic quality index ISCE is: to strengthen the geometric component and in particular the transformations in the plane. The research was qualitative and in particular research action. A pretest was applied in order to determine the level of student learning; Then, three classroom projects were designed, divided into 10 work sessions, according to the phases of the Van Hiele model and using Geogebra as a tool for ICT (information and communication technologies). In the process the learning and some of its characteristics were analyzed. The Van Hiele model facilitated the learning of translations in the plane and allowed a permanent evaluation of the learning process. Including TIC tools allowed students to take the step through the integration phase in a practical and enjoyable way. Context-based activities should be designed and teamwork encouraged.
\end{abstract}

Keywords: Learning, Plane displacements, Van Hiele model, Geogebra.

\title{
Resumo
}

No artigo, analisa-se o processo de aprendizagem das traduções no plano seguindo o modelo de Van Hiele e integração do software geogebra, nos alunos do sétimo ano do Centro Educativo Rural Sucre do município de Mutiscua, Colômbia. Estes alunos têm mostrado resultados baixos nos testes e entre as sugestões dadas pelo ministério no índice sintético de qualidade ISCE é: fortalecer o componente geométrico e em particular as transformações no plano. A pesquisa foi qualitativa e particularmente pesquisa-ação. Um pré-teste foi aplicado para determinar o nível de aprendizado do aluno; Em seguida, três projetos de sala de aula divididos em 10 sessões de trabalho foram projetados de acordo com as fases do modelo de Van Hiele e usando o Geogebra como uma ferramenta de TIC (tecnologias de informação e comunicação). Ao final, seu escopo foi avaliado por meio de um pós-teste. No processo, a aprendizagem e algumas de suas características foram analisadas. O modelo de Van Hiele facilitou a aprendizagem das traduções no plano e permitiu uma avaliação 
permanente do processo de aprendizagem. A inclusão de ferramentas de TIC permitiu que os alunos passassem pela fase de integração de maneira prática e agradável. As atividades baseadas no contexto devem ser projetadas e o trabalho em equipe deve ser incentivado.

Palavras-chave: Aprendizagem, Traduções de plano, modelo de Van Hiele, Geogebra.

\section{Introducción}

Para la realización del presente estudio, fue necesario hacer un análisis del desempeño que los estudiantes colombianos han mostrado al presentar pruebas internacionales, nacionales y locales. Se analizaron los resultados de las pruebas PISA en las cuales los estudiantes colombianos obtienen una clasificación bastante inferior a los de otros países como México y Chile, y están muy por debajo del promedio de los países que pertenecen a la Organización para la Cooperación y el Desarrollo Económico (OCDE).

En el ámbito local, los resultados del Centro Educativo Rural Sucre han estado por debajo de los resultados a nivel departamental y a nivel nacional. Además, en el área de matemática en el centro educativo sucre en el 2014, el 23\% de los estudiantes obtuvo niveles insuficientes, el $68 \%$ alcanza solo los niveles básicos, el 10\% resultados satisfactorios y no cuenta con estudiantes que logren niveles avanzados; mientras que en el año 2015 el porcentaje en insuficiente es de 7\%, el de mínimo 63\%, el de satisfactorio $20 \%$ y solo el $10 \%$ logra niveles avanzados.

Para obtener unos buenos resultados se debe reconocer que el componente geométrico tiene igual importancia que los demás procesos cognitivos, por lo cual es de gran relevancia desarrollar y fortalecer el aprendizaje en este aspecto, además, al analizar los resultados de las pruebas saber presentadas por los estudiantes de quinto grado en 2014 es este componente el que necesita mayor fortalecimiento.

Ahora bien, los resultados obtenidos por los estudiantes en 2015, en la prueba de matemáticas de quinto grado señalan que en la competencia de razonamiento el $71 \%$ de los estudiantes no conjetura ni verifica los resultados de aplicar transformaciones a figuras en el plano como lo señala el informe del índice Sintético de Calidad Educativa (ISCE) entregado en el 2016. Además, en noveno grado los resultados reflejaron que los estudiantes tienen dificultad para localizar, describir e identificar 
características de figuras en el plano.

Estos aspectos fueron los insumos básicos para realizar esta investigación. ¿Qué nivel de aprendizaje tienen los estudiantes de séptimo grado en cuanto a las traslaciones en el plano?; ¿Cómo se puede apoyar el proceso de aprendizaje de las traslaciones? ¿Qué beneficios se obtienen en el aprendizaje de las traslaciones del plano al implementar proyectos de aula basados en el modelo de van Hiele $y$ mediados por geogebra? son preguntas que podemos responder al finalizar este trabajo investigativo.

Después de estudiar las dificultades que se presentan en el área de matemáticas y al ver que la geometría es un pilar fundamental que hay que fortalecer, se optó por utilizar el método de Van Hiele para mejorar el aprendizaje. En palabras de Fouz \& De Donosti, (2001, p. 92). "el aprendizaje de la geometría se hace pasando por unos determinados niveles de pensamiento y conocimiento... que no van asociados a la edad... que sólo alcanzado un nivel se puede pasar al siguiente". Para conseguir que el estudiante pase de un nivel a otro superior, debe cumplir con una serie de fases de aprendizaje en las cuales siempre se debe partir de lo que el alumno sabe e iniciar su proceso de aprendizaje a partir de ahí. Esto es acorde con lo que piensa
Ausubel (1978) "Si tuviera que reducir toda la Psicología Educativa a un solo principio diría lo siguiente: el factor más importante que influye en el aprendizaje es lo que el alumno/a sabe. Averígüese esto y enséñese en consecuencia".

Razonablemente, el aprendizaje de la geometría apoyado en el modelo de Van Hiele, permite que los estudiantes reestructuren la información partiendo de sus conocimientos previos, percibiendo la información de primera mano y apoyándose en lo que hasta ese momento conocen, luego si se realiza una orientación bien estructurada que desequilibre la información existente. (Pozo, 1997).

Se desea subrayar que debe ir a la par con el avance tecnológico que hoy en día se está dando. Es evidente que para mejorar el aprendizaje en los estudiantes deben incluirse herramientas para la información y comunicación (TIC) como complemento en la labor pedagógica. Otros investigadores lo han observado como el caso de (Maldonado, 2013, p. 78).

Después de realizar el análisis de los resultados del pre y postest, con el software SPSS y el estadístico t-Studend, podemos observar, a nivel general, que el grupo en el 
que la intervención se basaba en el modelo de Van Hiele y el software Geogebra obtuvo la mayor variación positiva en el nivel de razonamiento 1 (Reconocimiento) y el nivel 3 (Clasificación).

También, (Vargas \& Gamboa, 2013, p. 114) expresan que:

Los estudiantes que usaron representaciones con software de geometría se sintieron más motivados a explorar, a plantear conjeturas y a probarlas, que aquellos estudiantes que usan representaciones tradicionales plasmadas con lápiz y papel. Los estudiantes expresaron que el uso del software les dio autonomía en el aula, les permitió explorar y aclarar sus dudas; además de ser algo novedoso que los sacó de la rutina del aula.

Estas son razones del porqué herramientas como el geogebra hacen más llamativas y motivantes las clases. Al mediar las TIC con el modelo pedagógico de Van Hiele los resultados en el aprendizaje mejoran.

A pesar de lo que expresa el (Ministerio de Educación Nacional, 2016, p. 252) quien supone basado en documentos de la Organización para la Cooperación y el Desarrollo Económicos (OCDE) que en la parte rural: "no existe evidencia de que las nuevas tecnologías puedan mejorar los resultados educativos de los estudiantes, ya que los profesores a menudo carecen de entrenamiento sobre el uso de la tecnología". Se considera esto más bien como una oportunidad de manifestar todo lo contrario mostrando unos mejores resultados.

Pero por otra parte, en lo referente a la integración de las TIC y en el caso en particular el software geogebra el (Ministerio Nacional de Educacion, 2013 , p. 16) expresa que los docentes juegan un papel fundamental desde las aulas de clase, y deben para mejorar en su práctica docente, realizar una incorporación de las TIC. Porque son los docentes quienes deben liderar un cambio positivo en cuanto al uso apropiado de la tecnología y conseguir que todas estas herramientas que tenemos hoy día, sean utilizadas para sacar el mayor provecho, en particular en el enriquecimiento de nuestra labor pedagógica.

La investigación se desarrolló en el Centro Educativo Rural Sucre en su sede principal, en el Municipio de Mutiscua del departamento de Norte de Santander. Es una institución ubicada en la vereda sucre del municipio la cual aplica modelos de educación flexibles como escuela nueva y posprimaria. 
Cabe aclarar que, esta propuesta no solo está diseñada exclusivamente para estudiantes de Centros Educativos Rurales y que se puede aplicar perfectamente en cualquier institución de carácter urbano.

Se han tomado referentes como Maldonado (2013) En la universidad de Chile realizó un trabajo de investigación titulado "Enseñanza de las simetrías con uso de geogebra según el modelo de Van Hiele" con una comparación entre un currículo de enseñanza tradicional, otro aplicando el modelo de Van Hiele y otro que además de aplicar el modelo de Van hiele también utiliza el software Geogebra. Al final concluye que las intervención basabas en el modelo de Van Hiele muestran mejores resultados que la del modelo tradicional.

Por otra parte, Vargas \& Gamboa (2013). En su investigación La enseñanza del teorema de Pitágoras: una experiencia en el aula con el uso del geogebra, según el modelo de Van Hiele de la universidad de costa rica presenta los resultados de su experiencia llevada a cabo con estudiantes de secundaria, respecto al tema del teorema de Pitágoras y su recíproco, apoyado con el uso del geogebra y en el modelo de razonamiento geométrico de Van Hiele haciendo una comparación entre el modelo tradición y el modelo propuesto, encontrando que es mucho más motivante para los estudiantes el trabajo con el software geogebra y logrando que estudiantes con bajos resultados se mostraran más interesados, competitivos y con mejores resultados académicos.

También en la revista Números (2010) en el artículo "Transformaciones en el plano utilizando software de geometría dinámica", presenta una experiencia de aula que muestra el estudio de las isometrías en el plano en la educación secundaria, utilizando software de geometría dinámica y da razones para usar este y otros softwares.

A nivel nacional Julio (2014) de la universidad Nacional de Colombia y en su disertación doctoral "Las transformaciones en el plano y la noción de semejanza" nos comparten una propuesta para la enseñanza de las transformaciones en el plano e iniciar el aprendizaje del concepto de semejanza desde una perspectiva constructivista apoyada en el software geogebra y en el modelo de Van Hiele. Concluye que: la geometría y en particular los conceptos relacionados con las transformaciones geométricas han mostrado ser importantes en el arte, la pintura, escultura, la arquitectura, danzas en coreografía (simetría) y música; también que es más significativo el aprendizaje de la geometría si se hace uso de recursos 
tecnológicos como software de geometría dinámica, pues estos ayudan a visualizar, verificar y finalmente desarrollar la comprensión de los conceptos y que el proceso de aprendizaje de los estudiantes debe basarse en su propia actividad creadora, en sus propios descubrimientos, en sus motivaciones, donde el rol del profesor es el de orientador.

Así mismo Ardila \& Villadiego (2016) en la universidad de Antioquia nos presentan en su trabajo de investigación "Comprensión de las razones trigonométricas en el marco del modelo de Van Hiele" cuyo objetivo es hacer un análisis de la comprensión que tienen los estudiantes en la razones trigonométricas en el grado decimo de una institución educativa del municipio Santa Rosa de Osos. Concluye que los estudiantes logran una comprensión de muchos conceptos geométricos a partir de elementos visuales porque que estos contribuyen al desarrollo del pensamiento abstracto lo cual es propio de la aplicación del modelo de Van Hiele.

Otro referente importante es el del trabajo que se muestra en el artículo del 3er Simposio Internacional y IV Coloquio Regional de Investigación Educativa y Pedagógica (2016). Donde la profesora María Fuentes Hernández, Magister en Educación de la Universidad de Córdoba en su artículo "Desarrollo de los niveles de razonamiento geométrico según el modelo de van hiele y su relación con los estilos de aprendizaje" evalúa la eficacia del modelo de Van Hiele en el avance en los niveles de razonamiento geométrico de los estudiantes de grado $7^{\circ}$ de una institución educativa oficial en Córdoba (Colombia) y su relación con los estilos de aprendizaje. Posterior a la aplicación de la secuencia didáctica los estudiantes lograron adquirir fácilmente los niveles 1 y 2 de Van Hiele. Al comparar los resultados entre grupos en el pos test, notamos que al grupo experimental le fue mejor en todos los niveles de razonamiento. Los estudiantes que avanzaron en los grados de adquisición de los niveles 1 y 2 fueron capaces de nombrar más de un atributo de regularidad, las respuestas a los ítems fueron sustentadas de manera formal y nombraron características importantes de los polígonos.

En nuestro ámbito local podemos encontrar a Villamizar (2016) en el artículo “construcción de escenarios virtuales mediante el uso de un software de geometría dinámica" un ejemplo sobre como el uso de la tecnología y en particular de un software de geometría dinámica, sirven como herramientas de apoyo que le permiten al docente proponer una nueva forma de actuación en el aula, y una alternativa al 
estudiante diferente a la de usar lápiz y papel, además, permiten la manipulación de los objetos geométricos, hacer pruebas de arrastre como mover puntos, rectas, modificar parámetros, visualizar ciertas representaciones que difícilmente puedan observarse en el tablero o sobre el papel. Concluye en su aporte que el uso de la tecnología puede ser un factor motivante en la enseñanza de las matemáticas. Sin embargo, la tecnología por sí misma no genera conocimiento, ni sustituye al profesor, por lo tanto, es necesario enmarcar las aplicaciones tecnológicas y en este caso los entornos virtuales, dentro de una didáctica que promueva una comprensión y construcción de los conceptos matemáticos.

También, Vergara \& Lazzo (2013) en su artículo "Exploración de conceptos geométricos con el uso de Geogebra en estudiantes de cuarto año de básica primaria", Cuyo objetivo es el de resaltar la importancia de la Geometría en la escuela y como la tecnología con el uso de softwares de geometría dinámica se convierten en herramientas para crear nuevas estrategias de enseñanza, mejorando así el aprendizaje de los conceptos geométricos en los estudiantes. Concluye que los estudiantes pueden explorar a fondo cada una de las características y elementos de las funciones en un ambiente dinámico y la práctica docente deja de ser monótona, también nos recomienda invitar a docentes, padres de familia e instituciones a ver a la tecnología como una herramienta de apoyo en el proceso de aprendizaje de las matemáticas.

Por último, el trabajo de Gualdrón \& Gutierrez (2007). En su artículo "Una aproximación a los descriptores de los niveles de razonamiento de Van Hiele para la semejanza. El cual tiene el objetivo de diseñar una unidad de enseñanza para la semejanza de polígonos basándose en dicho modelo y llegando a la conclusión de que la mayoría de estudiantes a los que se les aplicó esta unidad consiguieron alcanzar el segundo nivel de razonamiento. Además, propone el trabajo con figuras homotéticas utilizando el marco teórico abordado.

\section{Metodología}

La investigación se enmarca desde el enfoque de investigación cualitativa. Se hace un análisis acerca de ¿Cómo es el proceso de aprendizaje de las traslaciones en el plano en los estudiantes de séptimo grado en el CER Sucre de Mutiscua al implementar proyectos de aula basados en el modelo de Van Hiele y el software geogebra?, esto no puede ser resuelto simplemente analizando unos datos estadísticos, requiere conocer diversos aspectos que solo se pueden observar en el proceso de investigación cualitativa "esto es lo que ofrece 
la investigación cualitativa. Proporciona diferentes alternativas al investigador para tener un conocimiento más profundo de la situación en concreto que le permitirá resolver un problema” (Balcázar, Gonzalez, Gurrola, \& Moysén, 2006, p. 21).

Hay que mencionar, además a (Hernandez, Fernandez, \& Baptista, 2007, p. 10) quienes dan algunas características de la investigación cualitativa: los participantes en la investigación se comportan naturalmente como en su vida cotidiana; no se pretende manipular ni controlar las variables; la pregunta de investigación puede modificarse durante el proceso de investigación; la recolección de datos está fuertemente influida por la experiencia y participación $\mathrm{y}$ no es necesario analizar los datos numérica o estadísticamente.

Al mismo tiempo es investigación acción y se aborda desde Elliott, (1990, p. 24) uno de los principales autores la toma como una oportunidad para mejorar el quehacer pedagógico así lo afirma en su documento la investigación acción en educación:

La investigación-acción se relaciona con los problemas prácticos cotidianos experimentados por los profesores, en vez de con los "problemas teóricos" definidos por los investigadores puros en el entorno de una disciplina del saber. Puede ser desarrollada por los mismos profesores o por alguien a quien ellos se lo encarguen.

Durante el proceso se utilizaron para la recolección de información instrumentos como el diario de campo, se grabaron en video las sesiones y se registraron las observaciones del proceso. Con estas herramientas se realiza un análisis del proceso de aprendizaje en los estudiantes objeto de estudio.

Se inició abordando la problemática observada y revisando literatura acerca del tema. Se encontró que el modelo de Van Hiele es un modelo propuesto desde hace mucho tiempo en los lineamientos curriculares de matemáticas del ministerio de educación nacional. Además, como este modelo es adecuado al problema detectado se comenzaron a diseñar las actividades con base a dicho modelo.

Se elaboró un test para identificar el nivel de razonamiento que tienen los estudiantes. Después de analizar el test se elaboraron los proyectos de aula apoyados en el modelo de Van Hiele y que incluyeron actividades en geogebra.

El pretest se aplicó a los estudiantes de séptimo grado en 
el mes de agosto del 2016 y en septiembre se comenzaron a aplicar las sesiones de los proyectos, pero solo se alcanzaron a aplicar 5 de 10 sesiones en ese grado. Las que faltaron al iniciar el año lectivo 2017 se terminaron de aplicar cuando los estudiantes estaban en el grado octavo. Finalizando la intervención se realizó la evaluación pos test en la que la mayoría de estudiantes obtuvieron resultados exitosos.

En el año 2017 se cambiaron los estudiantes objeto de estudio. En enero, con el diagnóstico, los estudiantes de séptimo grado, se validaron y aplicaron los proyectos de aula. Durante este proceso de intervención también se diseñaron y validaron las actividades de evaluación.

Los estudiantes mejoraron en muchos aspectos. Sus trabajos después de implementar la propuesta son más limpios, esto debido al uso adecuado de las herramientas. El trabajo se desarrolló de una forma dinámica, en ocasiones se hacía de forma individual y en otras por equipos y siempre se notó que utilizar el software Geogebra se incrementó la motivación para desarrollar los trabajos propuestos.

La evaluación se hizo en forma continua, en todas las actividades se observó y valoró el trabajo hecho por los estudiantes y se les solicitó entregar los trabajos al finalizar la clase. Además, enviaron los trabajos elaborados en Geogebra por correo al profesor. Para finalizar este proceso, se hicieron dos evaluaciones de todo el tema trabajado en los proyectos de aula.

Al aplicar la propuesta se llevaron registros de observación en diarios de campo, en él se llevaban anotaciones del trabajo realizado en clase. La aplicación de la propuesta en los estudiantes de séptimo en 2016 fue más sencilla ya que se aplicó a un grupo que no era multigrado; mientras que en la aplicada en el grupo de séptimo en 2017, eran menos estudiantes pero el proceso de intervención fue más dificultoso debido a que el grado séptimo y el grado sexto estaban en una sola aula y se debían orientar los dos cursos a la vez. Para minimizar esta dificultad se realizaron grabaciones en video que posteriormente se revisaron. Haber desarrollado la propuesta en dos ocasiones permitió ir mejorándola y corregir algunos errores de la primera aplicación.

La propuesta se aplicó a los estudiantes del grado séptimo en el Centro Educativo Rural Sucre. En el año 2016 la población eran 21 estudiantes del grado séptimo y la muestra los mismos 21 estudiantes. No se terminó de implementar la propuesta en este grupo por cuestión de tiempo. En el año 2017 se inició con el grupo de séptimo grado. 7 estudiantes 
todos pertenecientes a la comunidad rural de los cuales, la población fueron los 7 estudiantes del grado séptimo y la muestra los mismos 7 estudiantes. De estos 3 son mujeres y 4 son hombres con edades entre los 10 y los 13 años.

Se realizó un trabajo de investigación basado en el respeto a cada una de las personas que se involucran en el proyecto. Se presentan datos fidedignos $y$ confiables sobre los hallazgos y resultados encontrados. Si se utilizan evidencias fotográficas, videos y producción textual, se hace bajo previo consentimiento informado, avalado y firmado por los padres de familia o quien haga las veces de representante legal de los menores participantes en la investigación. Los datos o pruebas recolectadas se toman con fines educativos y pedagógicos.

\section{Resultados}

El análisis de resultados se ha realizado mediante el proceso de triangulación y se contó con varias fuentes de información en nuestro caso el pretest, los diarios de campo, el pos test. Se realizó también el proceso de observación en las sesiones, luego se revisaron los videos grabados y se compararon con los lineamientos del modelo de van Hiele

Con el pretest se identifican los conocimientos que tienen los estudiantes y el nivel razonamiento en el que están, se elaboró acorde al modelo de Van Hiele y abordando la temática para trabajar en la propuesta. Se observa la claridad que tienen los estudiantes en cuanto a los conceptos de algunas figuras; determina características de lo que entienden los estudiantes por traslación; también el análisis que hacen al observar traslaciones y como trasladar figuras.

Se utilizó el diario de campo como herramienta en esta investigación. Este instrumento permite llevar un registro de las prácticas investigativas, mejorarlas, enriquecerlas y transformarlas. Se fortaleció con la observación de los videos grabados. La observación es una técnica de investigación de fuentes primarias, que necesita de una planeación para abordar un objeto de estudio a través del trabajo de campo, la práctica y la teoría se retroalimentan y hacen que los diarios adquieran cada vez mayor profundidad en el discurso y así, exista una relación recíproca entre práctica y teoría. Por una parte, la práctica es la fuente y la raíz del conocimiento de la teoría, pero a su vez, la teoría se orienta y sirve a la práctica, para que esta sea más eficaz. (Martinez, 2007, p. 77).

El diario de campo se llevó en todas las sesiones del proyecto, se registraron datos en las clases en lo referente al trabajo de los estudiantes, a sus apreciaciones y 
observaciones así como sus recomendaciones.

El proceso de observación se hizo lo más riguroso posible; el docente y los estudiantes participaron de manera natural, además se realizaron grabaciones en video de todas las sesiones con el fin de obtener mayor información o de tener en cuenta aquellas que no se hayan apuntado durante el proceso. Según: (Balcázar, Gonzalez, Gurrola, \& Moysén, 2006, p. 34) la observación es: "el proceso de contemplar sistemáticamente y detenidamente cómo se desarrolla la vida social, sin manipularla ni modificarla, tal cual ella discurre por sí misma"

Para finalizar de implementar la propuesta se usó un postest para evidenciar el nuevo nivel de razonamiento que tienen los estudiantes después de aplicados los tres proyectos de aula. En ellos se plantean ejercicios para identificar traslaciones y otros movimientos en el plano, así como sus características y las relaciones de dichos movimientos con situaciones reales. También se proponen ejercicios de ubicación en el plano y para realizar movimientos. Se analiza además, como realizan traslaciones y otros movimientos con regla y compás. Se observa si los estudiantes reconocen características de las trasformaciones en el plano y cómo aplican los conocimientos que adquirieron durante el proceso; asimismo, cómo utilizan las herramientas trabajadas en el transcurso del mismo. Los instrumentos utilizados, el pretest, las sesiones de cada proyecto de aula y el postest fueron validados por personas idóneas.

Posterior a su aplicación, para el análisis de los datos se elaboraron rejillas de triangulación, las cuales evidenciaron categorías como aprendizaje, evaluación y motivación; estas a su vez se relacionaron con el modelo de Van Hiele, las herramientas TIC como geogebra y el trabajo en equipo.

Al aplicar el pretest se observó que los estudiantes están en el nivel de reconocimiento del modelo de Van Hiele en cuanto al tema de traslaciones, ya que lo hacen teniendo en cuenta las relaciones de semejanza, además no notan las características de una traslación debido a que al solicitarles encerrar los dibujos que representaban traslaciones lo hicieron de forma errónea, también se observa que la pulcritud con que realizan sus trabajos es muy regular.

El análisis de este pretest, así como las recomendaciones que se dan en el reporte de las pruebas saber; fueron los insumos necesarios para diseñar los proyectos de aula de la propuesta pedagógica, además de esta información, se plantearon 
situaciones contextualizadas utilizando la estructura de las fases del modelo de Van Hiele y se incluyeron actividades con el software geogebra, se pueden observar y/o utilizar con fines netamente pedagógicos, de ninguna forma comerciales, están alojados en la dirección http://matematiksucre.wixsite.co $\mathrm{m} /$ inicio/curiosidades.

Al implementar la propuesta pedagógica, en las sesiones del primer proyecto de aula se apreciaron los siguientes aspectos:

- Los estudiantes estuvieron motivados, les gustaron los temas que relacionan la tierra y la geometría y en especial, la geometría y el universo; las actividades adecuadas con su contexto son apropiadas, fáciles de orientar y los estudiantes las elaboran con gusto.

- Estuvieron muy comprometidos al realizar trabajo en equipo, los estudiantes se apoyaron y se preguntaron entre ellos acerca de las dificultades que tenían, un factor muy importante para fortalecer el proceso de aprendizaje. La estructura que propone el modelo de Van Hiele fue adecuada al trabajo realizado, ya que las actividades diseñadas incluían actividades tanto individuales como grupales.

- También se observó que los estudiantes estaban apresurados por realizar la actividad en el computador, Cualquier actividad que involucre estas herramientas motiva los estudiantes, pero debe orientarse adecuadamente su uso. Si esto es así, se facilita el proceso de aprendizaje.

- La evaluación se realizó en forma continua y los estudiantes no se preocuparon por la calificación; se valoró el trabajo individual y el trabajo en equipo; se observó el trabajo de todos los estudiantes, la responsabilidad y el agrado con el cual participaron en las sesiones del proyecto.

- El trabajo que desarrollaron con las herramientas geométricas fue agradable aunque dispendioso para los alumnos, por lo que se aumentó el tiempo previsto para esta actividad.

- Durante la aplicación del segundo proyecto, algunos estudiantes tuvieron dificultad al representar coordenadas, así que fue necesario retroalimentar el tema. Se participó en un ambiente de respeto entre compañeros y con el docente. El trabajo en equipo permaneció siendo muy importante para facilitar el proceso de aprendizaje.

Ya se comenzó a ver que los estudiantes empiezan a establecer regularidades de forma natural, en el caso de los ángulos, establecen algunos tipos de relaciones como la de ángulos suplementarios y aunque no hacen un razonamiento formal del mismo, si notan que un 
ángulo llano se puede formar por dos ángulos que sumen $180^{\circ}$, lo cual indica un avance en el nivel de análisis de Van Hiele en estos aspectos. En definitiva, los estudiantes integraron todos los conceptos que hasta ahora habían venido aprendiendo y cada vez los utilizaron con más naturalidad.

La actividad con el software geogebra aclaró muchas de las dudas que tenían algunos estudiantes, en el programa se localizaba un punto y se podía ver la coordenada en la vista algebraica; este ejercicio permitió comparar los trabajos realizados a mano con los que se hicieron en el computador. Gracias a esto, se puede afirmar que, las actividades que involucran herramientas TIC favorecen el afianzamiento del aprendizaje y este proceso se realiza de forma amena $y$ motivadora. Así pues, terminar las sesiones reforzándolas con actividades en geogebra permite hacer un trabajo dinámico y agradable y se retroalimenta todo el proceso. La fase de integración se puede realizar empleando el software geogebra, proponiendo ejercicios que involucren todo lo trabajado en cada sesión sin incluir temáticas nuevas.

Finalizando la aplicación de la propuesta pedagógica durante el tercer proyecto de aula se nota que el modelo de Van hiele hace que los estudiantes sean cuidadosos en sus observaciones; integrando muchos de los conceptos y temas propuestos al realizar el proceso de aprendizaje en forma escalonada y de una manera natural. Para continuar con éxito en el proceso se han retroalimentado

permanentemente las actividades en el plano cartesiano y aunque parece un tema sencillo debe continuar fortaleciéndose.

La evaluación se realizó desde el comienzo de todas las sesiones, se observó el trabajo de cada estudiante así como los trabajos que entregaron, se valoraron sus aportes individuales y grupales, también la actitud que mostraron durante las actividades de la sesión. Esto facilitó el proceso de aprendizaje debido a que, los estudiantes ahora realizan las actividades con el fin de aprender y no por la calificación.

La actividad en geogebra se desarrolló con más facilidad, ya es reconocido su entorno en gran parte, también se identifican las herramientas y su utilidad con más propiedad; y para finalizar, en la actividad de aplicación se integraron al aprendizaje los conceptos trabajados desde la primera sesión.

En general el modelo de Van Hiele fortalece el trabajo individual y el trabajo en equipos; genera confianza y participación entre los estudiantes y permite que los estudiantes corrijan cuando se equivocan. Al tener que estar pendientes de las 
características de un objeto o un proceso, son capaces de ver cuando cometen errores.

También se ha conseguido que los estudiantes tengan un mejor manejo de herramientas como compas, reglas y escuadras. Sus representaciones gráficas ahora son más ordenadas y limpias, sus trazos son más delicados y precisos.

La actividad de crear teselaciones fue muy apreciada por los estudiantes, se observó que estuvieron atentos para desarrollar el proceso de una manera adecuada.

El postest permitió evidenciar que los estudiantes se ubican correctamente y elaboran figuras en el plano cartesiano; efectúan traslaciones, reflexiones, y rotaciones haciendo diseños cada vez más precisos; mejoraron el lenguaje refiendose a los movimientos en el plano y en especial a las traslaciones; reconocen características de los elementos de la geometría y de polígonos; tienen claridad en la ubicación de coordenadas en el plano cartesiano y las relacionan con situaciones reales; pueden dibujar polígonos regulares utilizando regla y compás y utilizan procedimientos para trazar rectas perpendiculares y paralelas; realizan teselaciones sencillas utilizando polígonos regulares y teselaciones un poco más complicadas usando simetrías, traslaciones $\mathrm{y}$ rotaciones.

Cabe recordar que el transcurso de las fases de Van hiele no es tan especifico y siempre debemos estar analizando que nos sirve $y$ que no en el proceso de aprendizaje para que nuestros estudiantes avancen apropiadamente.

Podemos señalar entre otras que, en la base del aprendizaje de la Geometría, hay dos elementos importantes "el lenguaje utilizado" y "la significatividad de los contenidos". Lo primero implica que los niveles, y su adquisición, van muy unidos al dominio del lenguaje adecuado $\mathrm{y}$, lo segundo, que sólo van a asimilar aquello que les es presentado a nivel de su razonamiento. Si no es así se debe esperar a que lo alcancen para enseñarles un contenido matemático nuevo. Para terminar estos previos Van Hiele señala que "no hay un método panacea para alcanzar un nivel nuevo pero, mediante unas actividades y enseñanza adecuadas se puede predisponer a los estudiantes a su adquisición". (Fouz \& De Donosti, Modelo de Van Hiele para la didactica de la geometria, 2001)

Pero sin duda, los resultados obtenidos evidencian que los estudiantes han subido de nivel en 
la escala de Van Hiele en el relativo a movimientos en el plano $\mathrm{y}$ en particular a en las traslaciones.

\section{Discusión y conclusiones}

Después de aplicar el pretest, los estudiantes reconocen las figuras de una forma global; describen el aspecto que ven de las figuras pero no notan características similares; no son capaces de generalizar las características que observan y en cuanto al tema de traslaciones, lo hacen teniendo en cuenta solo las relaciones de semejanza. Estos aspectos indican que los estudiantes están en el nivel 1 o de reconocimiento de Van Hiele.

Después de analizar el nivel de aprendizaje de los estudiantes de acuerdo al modelo de van hiele se concluyó que deben diseñarse las actividades de los proyectos de aula de acuerdo con las fases de Van Hiele incluyendo actividades apoyadas en geogebra teniendo en cuenta los temas y conceptos necesarios para abordar la temática de movimientos en el plano.

Durante la implementación de los proyectos se pueden destacar lo siguiente:

Es muy importante incorporar en nuestra labor pedagógica el diseño de actividades que partan del contexto en el cual el estudiante vive a diario ya que así se consigue que él esté motivado y aprenda de lo que hace, Las actividades que involucran el trabajo en equipo benefician el aprendizaje.

El articular al modelo de Van Hiele $y$ un software como Geogebra, se consigue que los estudiantes integren más fácilmente el conocimiento y puedan completar las cinco fases de aprendizaje requeridas para ascender en su nivel de aprendizaje. La fase de integración se puede realizar haciendo actividades en las cuales se utilice el Software Geogebra. Las actividades que involucran herramientas TIC favorecen el afianzamiento del aprendizaje y este proceso se realiza de forma amena y motivadora, además permite hacer un trabajo dinámico, agradable y se retroalimenta todo lo trabajado en cada sesión; los estudiantes pueden comparar los trabajos hechos a mano con los hechos en el programa.

La aplicación del modelo de Van hiele ha permitido evidenciar que el aprendizaje ha sido significativo ya que los estudiantes recuerdan y aplican todo lo que han trabajado en las anteriores sesiones.

Las fases de Van Hiele especialmente la fase 3 propone hacer una socialización de las características observadas y se notó que esta motiva la 
participación de los estudiantes, además entre ellos se colaboran cuando se presentan dificultades. El modelo de Van Hiele fortalece tanto el trabajo individual como el trabajo en equipos y genera confianza y participación entre los estudiantes.

En la medida en que se avanzó en el desarrollo de la propuesta, los estudiantes ya comienzan a establecer regularidades de forma más natural y están integrando todos los temas y conceptos trabajados, aunque no sea un razonamiento formal; también tienen un mejor manejo de herramientas como compas, reglas y escuadras. Sus representaciones graficas son más ordenadas y limpias después de la aplicación de la propuesta.

Los estudiantes reconocen Las sesiones diseñadas acorde al modelo de Van Hiele y tienen claridad en el trabajo durante las clases, aunque no se les mencionan las fases de aprendizaje, ellos saben que primero debe hacerse un proceso de reconocimiento y de identificación de características, luego deben compartir con sus compañeros y mejorar sus apreciaciones, al final realizar otras actividades que involucren afianzamiento $\mathrm{y}$ al terminar las actividades se realiza la practica con Geogebra.

El proceso evaluativo debe hacerse en forma permanente así los estudiantes desarrollan su proceso de aprendizaje de tal forma que no les preocupan las evaluaciones y realizan las actividades con el fin de aprender y no por la calificación.

Hay muchas actividades que como docentes creemos que pueden realizarse de forma rápida, es necesario analizar estas situaciones y si es el caso emplear más tiempo y más ejercicios que permitan hacer el proceso de aprendizaje más seguro y significativo, no debe considerarse como pérdida de tiempo.

Los procesos de aprendizaje donde se incluyen herramientas TIC no solo en geometría son motivantes para los estudiantes, sino que pueden ser aplicados en otros procesos de aprendizaje en los cuales sea posible, este hecho muchas veces solo depende de la creatividad que tenga el maestro para integrarlos.

Se recomienda implementar el modelo de Van Hiele en los procesos de aprendizaje que tengan relación con el componente geométrico, ya que es una propuesta adecuada y que permite que todos los estudiantes aprendan sin importar el nivel en el que se encuentren, solo se debe partir del reconocimiento que ellos tienen.

Se recomienda la utilización de la página WIX http://matematiksucre.wixsite.co 
m/inicio/curiosidades. Que se elaboró durante el proceso de investigación en esta se encuentra la propuesta pedagógica completa y puede ser utilizada fines educativos, no comerciales.

\section{Referencias}

Ministerio Nacional de Educacion. (2013). Competencias TIC para el Desarrollo Profesional Docente. Bogotá: Oficina de Innovación Educativa. p. 16.

Ardila, D., \& Villadiego, K. (2016). Comprension de las razones trigonometricas en el marco del modelo educativo de Van Hiele. tesis para maestria. Medellín, Colombia. $172 \mathrm{p}$.

Balcázar, P., Gonzalez, N., Gurrola, G., \& Moysén, A. (2006). Investigacion Cualitativa. México: Universidad Autonoma de México. 241.

Elliott, J. (1990). La investigación acción en educacion. Morata, S. L. p. 24

Escobar, L., \& Guerra, S. (2015). Razonar geometricamente sobre lahiperbola en el marco del modelo de Van Hiele apoyados en el uso de Geogebra . tesis para maestria. Medellín, Colombia.

Fouz, F., \& De Donosti, B. (2001). Modelo de Van Hiele para la didactica de la geometria.
Fouz, F., \& De Donosti, B. (2001). Modelo de Van Hiele para la didactica de la Geometria.

Gualdrón, E., \& Gutierrez, A. (2007). Una aproximacion a los descriptores de los niveles de razonamiento de Van Hiele para la semejanza. 370-380.

Hernandez, R., Fernandez, C., \& Baptista, P. (2007). Metodologia de la investigacion. México: McGraw Hill.

Julio, L. (2014). Las transformaciones en el plano y la nocion de semejanza. Trabajo de grado para maestria. Bogotá, Colombia.

Maldonado, L. (2013). Enseñanza de las Simetrias con unso de Geogebra segun el modelo de Van Hiele . Santiago.

Martinez, L. (2007). La observación y el diario de campo en la definición de un tema de investigación. perfiles libertadores. Bogotá. pp.73-80.

Ministerio de Educación Nacional. (2016). La educación en Colombia. Paris: ocde.

Pozo, J. I. (1997). Teorias Cognitivas del aprendizaje. Madris: Ediciones Morata, S.L.

Vargas, G., \& Gamboa, R. (2013). La enseñanza del teorema de pitágoras: una experiencia en el aula con el uso del geogebra, 
según el modelo de van hiele. uniciencia, 95-118.

Vergara, L., \& Lazzo, L. (2013). Exploración de conceptos geométricos con el uso de. VIII Simposio Nororiental de Matemáticas Universidad Industrial de Santander, 336340.

Villamizar, f. (2016). construcción de escenarios virtuales mediante el uso de un software de geometría dinámica. Encuentro internacional en educación, 166-170. 


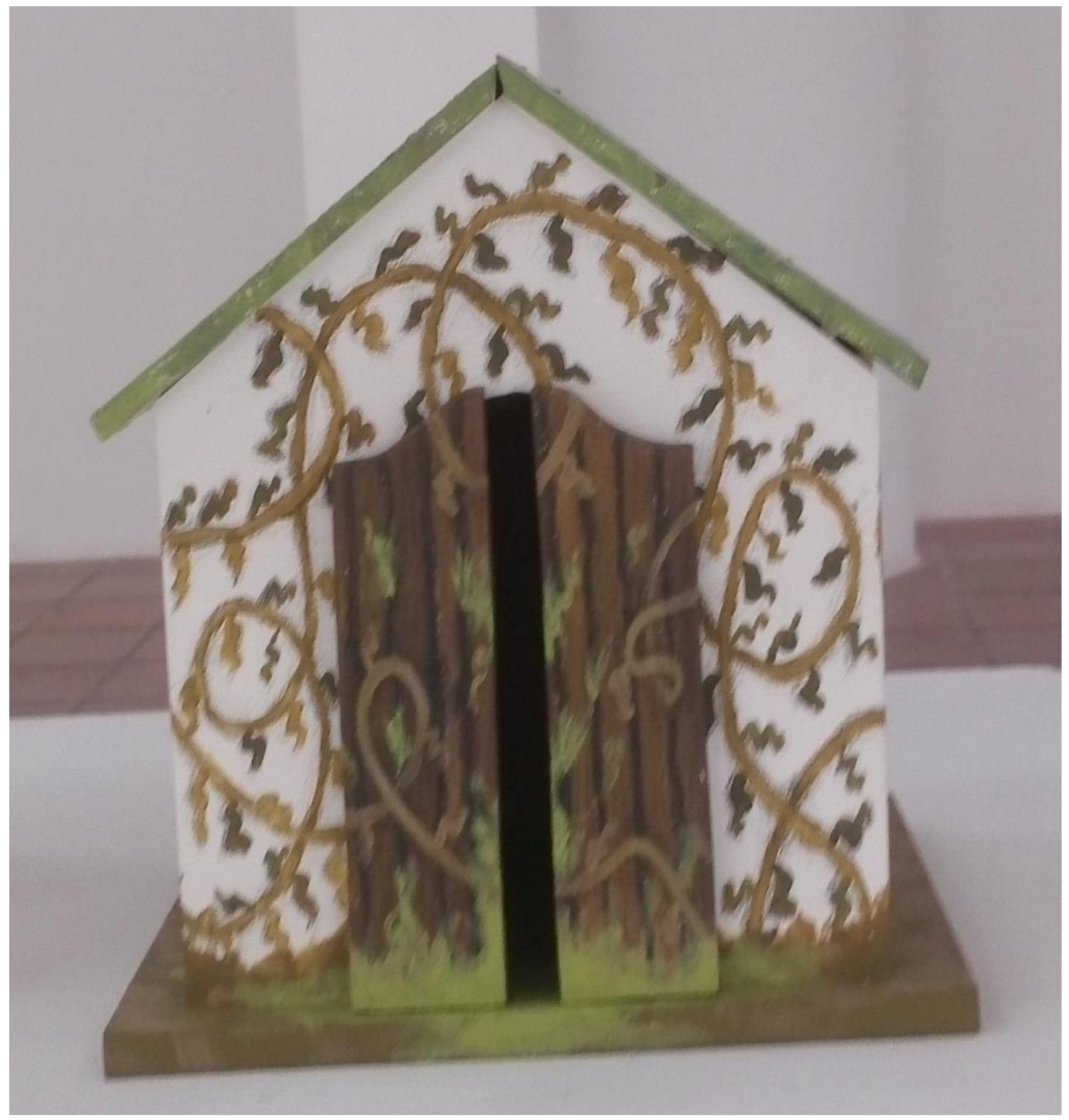

Obra: Chamiza

Autor: Valentina García T.

Técnica: Acrílico - Vinilo

Territorio: Neiva 